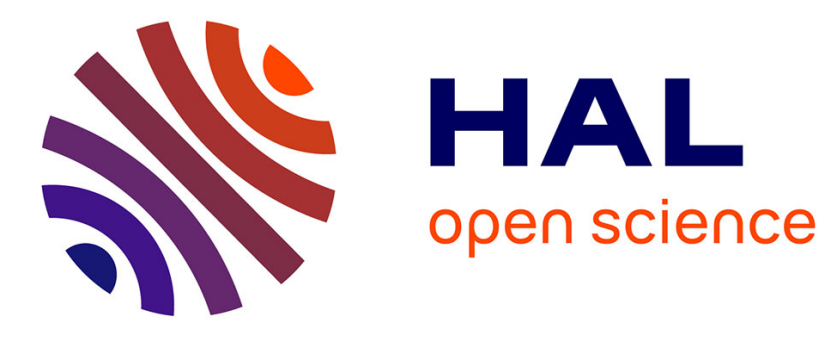

\title{
2D Hybrid Yttrium Iron Garnet Magnetic Sensor Noise Characterization
}

\author{
Basile Dufay, Sébastien Saez, Christophe Cordier, Christophe Dolabdjian, \\ Christian Dubuc, E. Hristoforou, S. Ubizskii
}

\section{- To cite this version:}

Basile Dufay, Sébastien Saez, Christophe Cordier, Christophe Dolabdjian, Christian Dubuc, et al.. 2D Hybrid Yttrium Iron Garnet Magnetic Sensor Noise Characterization. IEEE Sensors Journal, 2011, 11 (12), pp.3211-3215. hal-00986946

\section{HAL Id: hal-00986946 https://hal.science/hal-00986946}

Submitted on 5 May 2014

HAL is a multi-disciplinary open access archive for the deposit and dissemination of scientific research documents, whether they are published or not. The documents may come from teaching and research institutions in France or abroad, or from public or private research centers.
L'archive ouverte pluridisciplinaire HAL, est destinée au dépôt et à la diffusion de documents scientifiques de niveau recherche, publiés ou non, émanant des établissements d'enseignement et de recherche français ou étrangers, des laboratoires publics ou privés. 


\title{
2D Hybrid Yttrium Iron Garnet Magnetic Sensor Noise Characterization
}

\author{
B. Dufay, S. Saez, C. Cordier, C. Dolabdjian, C. Dubuc, E. Hristoforou, and S. Ubizskii
}

\begin{abstract}
This paper deals with the noise characterization of a magnetic field hybrid sensor based on flux-gate-like magnetometer. In the used layout, a magnetic core, like an Yttrium-Iron-Garnet (YIG) thin film, is driven to saturation by a rotating magnetization field, which induces a modulated magnetic field. The latter is sensed, by means of one or more punctual sensors, as an image of the applied magnetic field vector components.

Both theoretical principles and main equivalent magnetic noise sources are explained. Sensor performance is given and compared with measurements. The experimental setup and limiting factors, such as the coupling factor between the magnetization and the sensed signal, are also evaluated and analyzed. That leads to the illustration of the system performance in term of noise and sensitivity.
\end{abstract}

Index Terms-Magnetometer, noise, YIG.

\section{INTRODUCTION}

$\mathbf{T}$ HE presented device is based on a combined flux-gate and Hall sensor arrangement. The sensor principle is similar to a previous work based on 3D flux-gate sensor [1], which does not illustrate the corresponding sensor performance in details. Furthermore, the mentioned sensor and the 2D or 3D field sensing ability was not completely described in that work. Also, noise measurements were reported by Valeiko et al. in an abstract in the EMSA 2000 Conference [2]. However, this work was never published in a journal paper and/or elaborated by another research group until now. These facts have been the motivation to expose the performance of the device presented in this work in terms of noise and sensitivity, as well as to show device improvement, compared to a bare integrated sensor.

In flux-gate sensors, a limiting factor of noise performance is mainly due to Barkhausen jumps [3]. This drawback appears in magnetic materials due to domain wall motion. A way to elim-

B. Dufay, S. Saez, C. Cordier, C. Dolabdjian, and C. Dubuc are with the Groupe de Recherche en Informatique, Image, Automatique et Instrumentation de Caen (GREYC), CNRS UMR 6072 - ENSICAEN and the University of Caen, Caen Cedex, 14050 France (e-mail: C.Dolabdjian@ greyc.ensicaen.fr).

E. Hristoforou is with the Department of Mining and Metallurgical Engineering, Section of Metallurgy and Technology of Materials, Laboratory of Physical Metallurg, National Technical University of Athens, Athens 15780 Greece.

S. Ubizskii is with the R\&D Center of Solid State Electronics and Sensors, Lviv Polytechnic National University, Lviv, 79013, Ukraine.

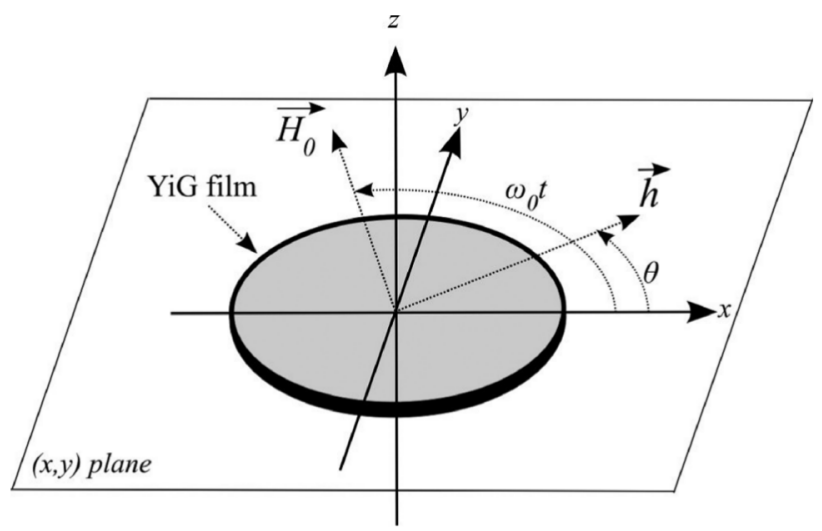

Fig. 1. Sketch view of applied in-plane fields on the thin YIG core.

inate the Barkhausen jumps is to keep quasi-continuously the material in deep saturation state. This can be achieved by applying a rotating excitation field on a soft magnetic material as an Yttrium-Iron-Garnet (YIG) thin film [1], [4]. The given magnetometer allows for 2D magnetic field measurements through a single device. It seems also suitable for a single chip design.

This paper is organized as follows. The theoretical magnetic sensor principle of operation is presented in Section II. Section III is dedicated to the sensor characteristics. Experimental results are given in Section IV which is followed by conclusions.

\section{PRINCIPLE OF Operation}

As given in Fig. 1, a rotating magnetic field $\mathbf{H}_{\mathbf{0}}$ rotates at the angular frequency $\omega_{0}$ and it is applied on the magnetic YIG thin-film plane. Its in-plane components are given by

$$
\left\{\begin{array}{l}
H_{0 x}=H_{0} \cos \left(\omega_{0} t\right) \\
H_{0 y}=H_{0} \sin \left(\omega_{0} t\right) \\
H_{0 z}=0
\end{array}\right.
$$

where $H_{0}$ is the rotating magnetic field vector amplitude. The quasi-static magnetic field $\mathbf{h}$, which is to be measured, is applied in the YIG film plane (cf. Fig. 1), its components being

$$
\left\{\begin{array}{l}
h_{x}=h \cos (\theta) \\
h_{y}=h \sin (\theta) . \\
h_{z}=0
\end{array} .\right.
$$

The resulting field $\mathbf{H}_{\mathbf{T}}$ is thus given by the superposition of both fields its components being

$$
\left\{\begin{array}{l}
H_{T_{x}}=H_{0} \cos \left(\omega_{0} t\right)+h \cos (\theta) \\
H_{T_{y}}=H_{0} \sin \left(\omega_{0} t\right)+h \sin (\theta) \\
H_{T_{y}}=0
\end{array} .\right.
$$


If the amplitude of $\mathbf{H}_{\mathbf{T}}$ is larger than $H_{S}$, the saturation field amplitude of the YIG film, the material is continuously saturated. The magnetization amplitude $\mathbf{M}$ has an angular direction which follows that of $\mathbf{H}_{\mathbf{T}}$ and its amplitude is approximately equal to the saturation value $M_{S} . \mathbf{M}$ is given by

$$
\left\{\begin{array}{l}
M_{x}=M_{S} \cos (\phi(t)) \\
M_{y}=M_{S} \sin (\phi(t)) \\
M_{z} \approx 0
\end{array}\right.
$$

where

$$
\phi(t)\left(=\arctan \left(\frac{H_{0} \sin \left(\omega_{0} t\right)+h \sin (\theta)}{H_{0} \cos \left(\omega_{0} t\right)+h \cos (\theta)}\right)\right)
$$

is the angular direction of $\mathbf{H}_{\mathbf{T}}$. Considering that $\mathrm{h} \ll \mathrm{H}_{0}$ and the associated Taylor series of $\phi(t)$, the thin YIG film magnetization can be rewritten as

$$
\left\{\begin{array}{l}
M_{x}=M_{S} \cos \left(\omega_{0} t-\frac{h}{H_{0}} \sin \left(\omega_{0} t-\theta\right)\right) \\
M_{y}=M_{S} \sin \left(\omega_{0} t-\frac{h}{H_{0}} \sin \left(\omega_{0} t-\theta\right)\right) \\
M_{z} \approx 0
\end{array} .\right.
$$

A punctual magnetic sensor is placed accordingly to measure the field exiting the thin film along the $x$-direction. It senses a magnetic field $B_{\text {induced }}$ which can be written as $B_{\text {induced }}=k M_{x}$, where $k(\mathrm{H} / \mathrm{m})$ is a coupling factor between the internal YIG thin-film magnetization $x$-component value and the external field induced at the sensor. Considering the punctual magnetic sensor sensitivity as $T_{H}$ (in $\mathrm{V} / \mathrm{T}$ ), $h$ $\ll H_{0}$ and noticing that a sinusoidal signal can be represented with classical Jacobi-Anger expansion associated to Bessel functions, the output signal, $V_{H}$, equals to

$$
V_{H} \approx T_{H} k M_{S}\left[\cos \left(\omega_{0} t\right)+\frac{h}{2 H_{0}}\left\{\cos (\theta)-\cos \left(2 \omega_{0} t-\theta\right)\right\}\right] \text {. }
$$

By using an in-phase and quadrature lock-in detection at twice the frequency $f_{0}$, we are able to obtain both amplitude, $h$, and in-plane direction $\theta$ of the external applied quasi-static field $\mathbf{h}$ or quasi-static induction field $\mathbf{b}\left(\mathbf{b}=\mu_{0} \mathbf{h}\right)$. After the lock-in detections, the sensitivity, $T_{r}$ (in $\mathrm{V} / \mathrm{T}$ ), of the hybrid device, versus $b$, can be evaluated as

$$
T_{r} \approx \frac{k M_{s}}{2 \mu_{0} H_{0}} T_{H}=G T_{H}
$$

where $G$ represents the hybrid sensor sensitivity improvement term compared to a punctual magnetometer. In order to increase $G$ as much as possible, an optimal coupling factor $k$, or $M_{s} / H_{0}$ ratio is required [4].

\section{SENSOR CHARACTERISTICS}

\section{A. Magnetization Process of the YIG Core Material}

The core element is made in the form of disk of around $1 \mathrm{~cm}$ in diameter from the epitaxial YIG film grown by liquid phase epitaxy on a nonmagnetic Gadolinium Gallium Garnet (GGG) substrate of (111) orientation.

The YIG thin film is a single crystal with a thickness in the order of $\mu \mathrm{m}$. It possesses a mixed magnetic anisotropy that is a combination of the magnetic crystallographic anisotropy, the

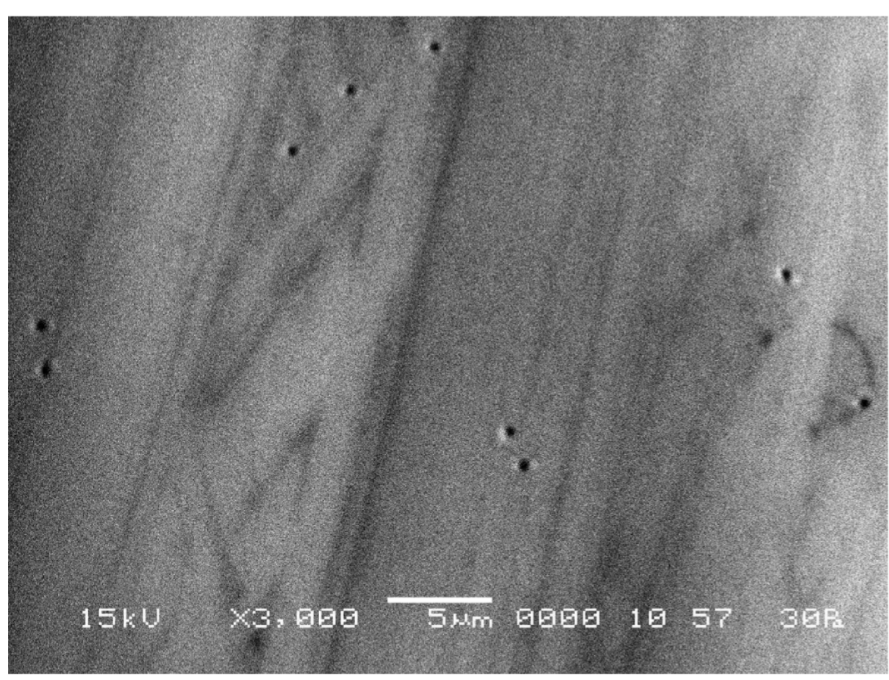

Fig. 2. SEM micrograph of YIG film after etching, illustrating no presence of grains and presence of imperfections in the form of randomly oriented holes.

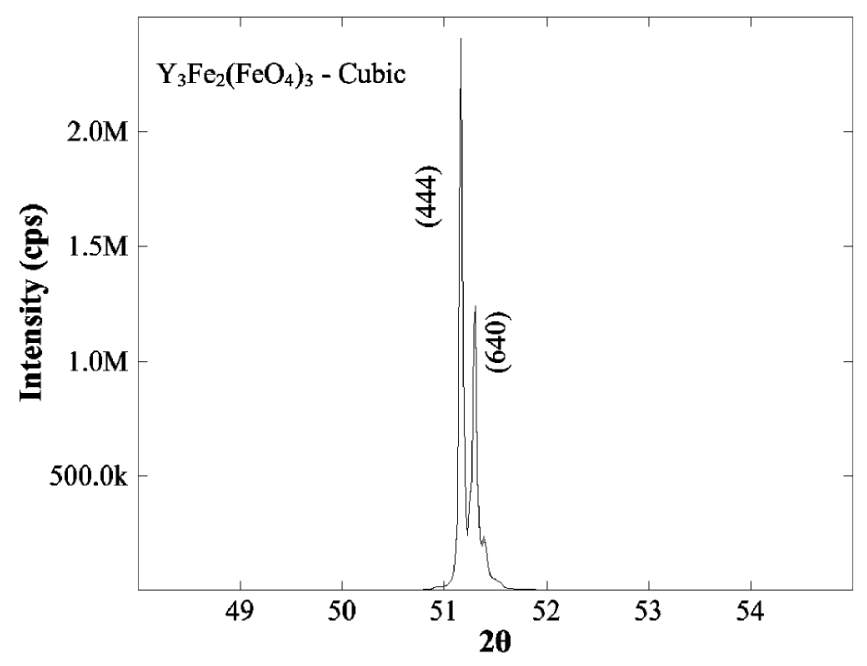

Fig. 3. Single crystal XRD response concerning the YIG film.

moderate perpendicular induced uniaxial magnetic anisotropy, and the shape anisotropy of the film. The easy magnetization axes of the sensing element do not lie in the sensing element plane but deviate from it for some angle [5].

A typical scanning electron microscope (SEM) back scattering micrograph after chemical etching is shown in Fig. 2, illustrating absence of grains and occasional-random presence of holes. A X-ray diffraction (XRD) response of the YIG thin film is given in Fig. 3, illustrating the behavior of a single crystal, by means of high single peak response in the order of $2.5 \mathrm{Mcps}$ and negligible half width height. The XRD response illustrates two main anisotropic orientations namely the (111) out of plane anisotropy and smaller in-plane anisotropy. Both allow for different magnetic response. The presence of randomly oriented holes in the film, suggests possible pinning centers during rotation of magnetization.

Therefore, when this film is subjected to external rotational magnetic film its magnetization vector may follow two paths: one rotational in-plane and one following the magnetic crystallographic easy axes [1]. The unidirectional magnetic response 


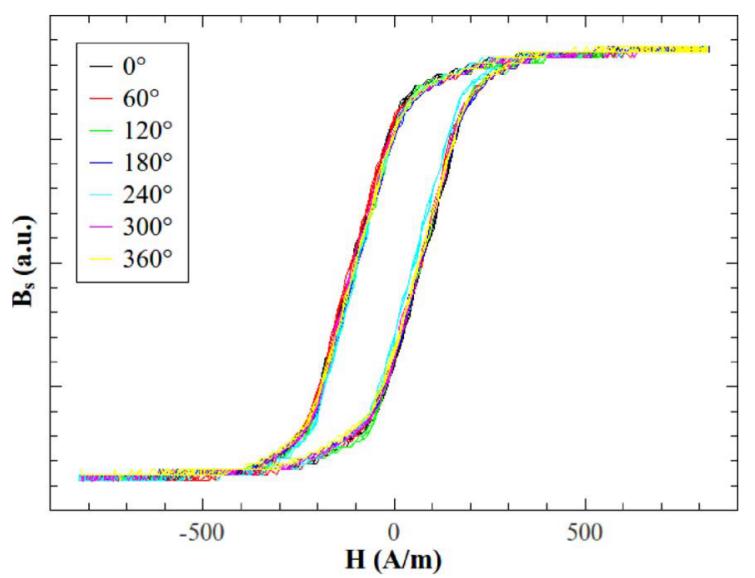

Fig. 4. In-plane magnetization component response in different angles $\theta$ corresponding to the easy axes. Here, the applied field frequency is $1 \mathrm{kHz}$.

on the easy axes is illustrated in Fig. 4 where no difference between the magnetization curves is observed as expected.

\section{B. Noise Sources}

1) Intrinsic Magnetic Sensor Noise Induced by the YIG Core: The use of the described rotating magnetization excludes the magnetic noise from Barkhausen jumps. Nevertheless, it is still available some noise sources due to thermal fluctuations in the material. The fluctuation-dissipation theorem and the known thermodynamic relations can be used to evaluate the intrinsic magnetic core noise spectral density. As evaluated by Vetosko et al. [7], that intrinsic noise at the excitation angular frequency $\left\langle b_{n}\right\rangle_{\omega_{0}}$ is given by

$$
\left\langle b_{n}\right\rangle_{\omega_{0}} \approx \sqrt{\frac{T k_{B} \Delta H}{4 \pi V \gamma M_{s}^{2}}}
$$

where $T$ is the absolute temperature, $k_{B}$ the Boltzmann constant, $V$ the magnetic film volume, $\Delta H$ the ferromagnetic resonance line-width, and $\gamma$ the gyromagnetic ratio. With $T=$ $300 \mathrm{~K}, \mathrm{~V}=2.510^{-10} \mathrm{~m}^{3}, \Delta H=79.6 \mathrm{~A} / \mathrm{m}, \gamma / 2 \pi=$ $28 \mathrm{GHz} / \mathrm{T}$ and $M_{s}=11.2 \mathrm{kA} / \mathrm{m}$, a numerical evaluation for a thin YIG film yields an intrinsic magnetic equivalent core noise spectral density of $2.2 \mathrm{fT} / \sqrt{\mathrm{Hz}}$.

2) Rotating Excitation Field Equivalent Noise Source: In practice, the magnetization loop of the YIG core is non-ideal. Near to the operating point, it can be approximated by a $\chi$-slope straight line, as shown in Fig. 5. In this case, the system performance is weakened by the excitation field noise. The saturation magnetization, $M_{s}$, and the sensed field, $h$, must be substituted in (6) by $M_{s} \approx \chi_{0} H_{0}+\chi h_{0}(t)$ and $h+h_{0}(t)$, respectively, where $h_{0}$ is the noise level of the excitation field, $\chi_{0}$ is the initial ratio $M_{s} / H_{0}$ and $\chi$ is the slope at the working point $H_{0}$. So, by neglecting the phase noise, the punctual magnetic sensor output may be rewritten as

$$
\begin{aligned}
& V_{H}=T_{H} k\left\{\chi_{0} H_{0}+\chi h_{0}(t)\right\} \\
& \quad\left\{\cos \left(\omega_{0} t\right)+\left(\frac{h+h_{0}(t)}{2 H_{0}}\right)\left[\cos (\theta)-\cos \left(2 \omega_{0} t-\theta\right)\right]\right\} .
\end{aligned}
$$

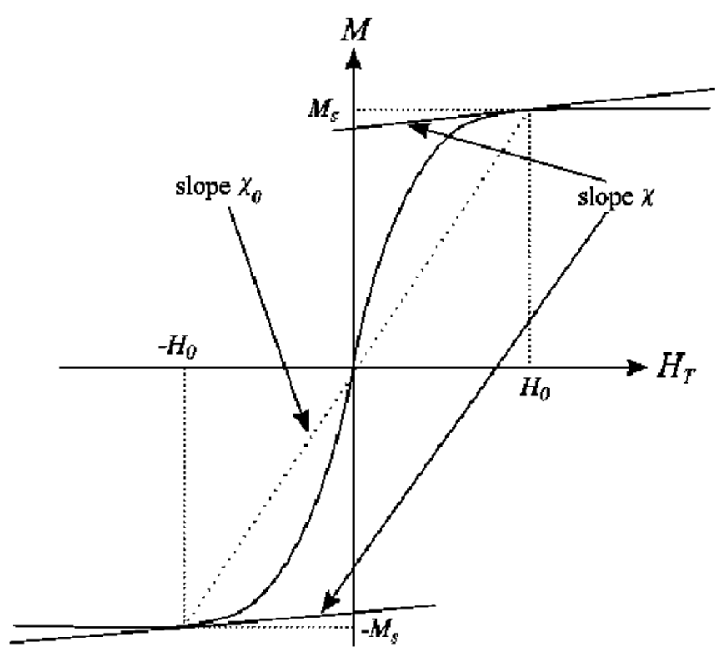

Fig. 5. Non-ideal magnetization process of the YIG film. This curve may be approximated by a $\chi$-slope straight line near to the operating point $\left(H_{0}, M_{s}\right)$.

Furthermore, the Signal to Noise Ratio (SNR) of the system (in terms of amplitude of detection), $S N R$, is limited to the generating rotating excitation field dynamic range as $S N R=\left(\chi_{0} H_{0} / \chi h_{0} \approx B_{\max } / b_{0}\right)$, where $B_{\max }$ is the highest detectable magnetic field induction.

3) Electronic Noise Source: The output voltage noise spectral density level of the punctual magnetic sensor, $e_{n}$, has to be considered, too. It is due to both intrinsic device in use and conditioning circuitry. This noise part appears directly on the output signal and can be considered, also, as an equivalent input magnetic noise.

So, demodulated output signal $V_{H_{-} d e m}(t)$ including all noise sources becomes

$$
\begin{aligned}
& V_{H_{\_} d e m}(t)=\frac{k M_{s}}{2 \mu_{0} H_{0}} T_{H} \\
& \qquad\left\{b+b_{n}(t)+\mu_{0} h_{0}(t)+\frac{\chi h_{0}(t)}{M_{s}} b+\frac{2 \mu_{0} H_{0}}{T_{H} k M_{s}} e_{n}(t)\right\}
\end{aligned}
$$

where $b$ is the sensed induction field, while the $\chi h_{0}(t) b_{n}(t)$ term has been neglected.

\section{COUPLING FACTOR}

The coupling factor $k$ is an important parameter to assess the intrinsic performance of the system. In our configuration, the punctual sensor is placed in such a way to sense the optimal vertical component (perpendicular to the $(x, y)$ plane) of the induced field. $k$ strongly depends on the punctual sensor position near the YIG film edge.

At first, $k$ has been evaluated with Finite-Element Method software $\mathrm{COMSOL}^{\circledR}$ and compared to measurements made by using a Hall sensor as punctual device [8]. Both measured and simulated results are presented in Fig. 6. According to this figure, the optimal sensor position is to be just over the edge of the YIG film, as near as possible to the surface. For each horizontal distance from the edge of the YIG film $d_{\text {edge }}$, an optimal Hall sensor position in the $z$ axis occurs. Even for this position, the value of $k$ is relatively low and degrades the performance of the system. As an example, for an internal magnetization 


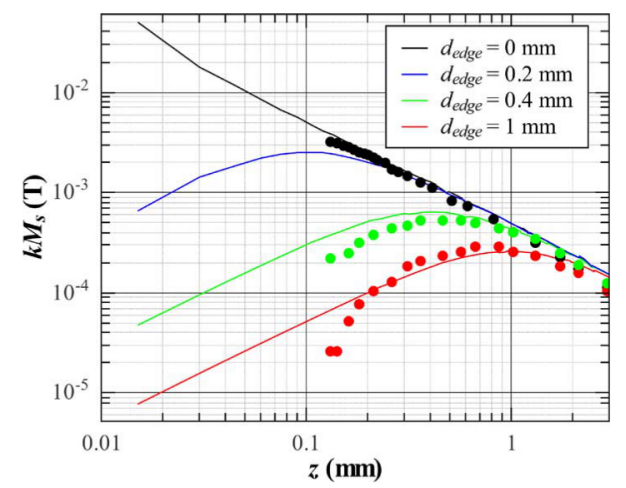

Fig. 6. Simulated (line) and measured (scatter) behavior of the induced magnetic field vertical component amplitude variation versus punctual sensor position $\left(0, r-d_{\text {edge }}, z\right)$ where $z$ represents the vertical distance of the sensor from the YIG film surface, $r$ is the YIG film radius and $d_{\text {edge }}$ represents the horizontal distance from the edge of the YIG film (axes are defined on Fig. 1). We note an expected $1 / z$-like decreasing. For the smallest values of $z$, the difference between experiment and simulation may be explained by Hall sensor positioning error. Simulation results have been obtained from finite element method software (a $20 \mathrm{~mm}$ diameter and $25 \mu \mathrm{m}$ thick ferromagnetic film has been modeled, having a constant in-plane magnetization of $100 \mathrm{kA} / \mathrm{m}$ to mimic the YIG thin-film saturated state).

value of $100 \mathrm{kA} / \mathrm{m}$ (as simulated), the theoretically-calculated magnetic field, induced at the sensor is about $125 \mathrm{mT}$.

Nevertheless, the maximum experimentally sensed value of $B_{\text {induced }}=k M_{s}$ is about $3 \mathrm{mT}$. With such value of $k M_{s}$, and considering an applied magnetic field amplitude, $H_{0}$, of $800 \mathrm{~A} / \mathrm{m}$, able to saturate the material, the sensitivity improvement factor $G$, defined in (7) is just around 1.5.

\section{REsults}

The rotating excitation field $\mathbf{H}_{\mathbf{0}}$ is applied with two perpendicular Helmholtz coils driven with sine and cosine currents, respectively. The sensing core magnetization is measured by a Hall sensor. In order to evaluate the sensitivity of the device, the external magnetic field $\mathbf{h}$ is applied as follows

$$
\left\{\begin{array}{c}
h_{x}=h \cos (\omega t) \\
h_{y}, h_{z}=0
\end{array}\right.
$$

with $h \ll H_{0}$ and $\omega \ll \omega_{0}$.

Futhermore, the demodulated output Hall signal is a classical Amplitude Modulation (AM) without carrier. Therefore, we have experimentally deduced the hybrid sensor sensitivity, $T_{r}$, by measuring and analyzing spectral line amplitudes. At first, some measurements have been made using an encapsulated Hall effect device, Honeywell ss496a1, having an intrinsic sensitivity $T_{H}$ of $25 \mathrm{~V} / \mathrm{T}$. The full system sensitivity $T_{r}$ was measured for a rotating excitation frequency $f_{0}\left(=\omega_{0} / 2 \pi\right)$ of $2 \mathrm{~Hz}$. Results are summarized in Table I. It illustrates the measured and the theoretical evaluation of the sensitivity improvement ratio $G$ as a function of the rotating excitation field amplitude $B_{0} . k M_{s}$ could be evaluated from the B-H loop of the thin YIG film for each given $B_{0}$. The theoretical values are deduced from (7). The agreement between theory and experiment validates the working principle of the device.

Experimentally, the most limiting part of noise in the system arises from the Hall sensor noise. By only considering the white
TABLE I

EVALUATION OF THE SENSITIVITY IMPROVEMENT RATIO

\begin{tabular}{|c|c|c|c|}
\hline \multirow{2}{*}{$\begin{array}{c}B_{0} \\
(\mu \mathrm{T})\end{array}$} & \multirow{2}{*}{$\begin{array}{c}|c| \\
(\mu \mathrm{T})\end{array}$} & \multicolumn{2}{|c|}{$G$} \\
\cline { 3 - 4 } & & Theoretical values & Measured values \\
\hline 180 & 500 & 1.40 & 1.68 \\
\hline 325 & 700 & 1.08 & 1.12 \\
\hline 720 & 780 & 0.52 & 0.56 \\
\hline
\end{tabular}

TABLE II

Sensor Performance. $B_{0}$ Is the Rotating Excitation Field Amplitude, $f_{0}$ Is the Rotating Excitation Field FreQuency, $k M_{s}$ IS THE SENSEd MAGNETIZATION, $G$ Is THE Sensitivity IMPROVEMENT, $b_{n}(f)$ Is THE EXPeCted Equivalent Magnetic Noise Spectral Density

\begin{tabular}{|c|c|c|c|c|c|}
\hline $\begin{array}{c}B_{0} \\
(\mathrm{mT})\end{array}$ & $\begin{array}{c}f_{0} \\
(\mathrm{kHz}]\end{array}$ & $\begin{array}{c}k M_{s} \\
(\mathrm{mT})\end{array}$ & $G$ & $\begin{array}{c}b_{n}(f) \\
(\mathrm{nT} / \sqrt{\mathrm{Hz}}]\end{array}$ & $\begin{array}{c}\left.b_{n}(f)\right|_{f=10 \mathrm{~Hz}} \\
(\mathrm{nTT} / \sqrt{\mathrm{Hz}})\end{array}$ \\
\hline $\begin{array}{c}\text { Bare Hall } \\
\text { sensor }\end{array}$ & - & - & 1 & $\sqrt{\left(\frac{8000}{\sqrt{f}}\right)^{2}+16^{2}}$ & 2600 \\
\hline 1 & 40 & 2 & 1 & 16 & 16 \\
\hline $0.18^{*}$ & 40 & 1.1 & 3 & 5.3 & 5.3 \\
\hline
\end{tabular}

$\left.{ }^{*}\right)$ in that case, magnetic field induction amplitude is not sufficient to saturate the thin YIG film.

noise frequency and the low-frequency bands, the Hall sensor equivalent magnetic power noise spectral density (PSD) is estimated by

$$
\operatorname{PSD}=\frac{\alpha_{n}^{2}}{f}+\beta_{n}^{2}
$$

with $\alpha_{n}=8 \mu \mathrm{T} / \sqrt{ } \mathrm{Hz}$ and $\beta_{n}=16 \mathrm{nT} / \sqrt{ } \mathrm{Hz}$. As explained in [9], the useful spectrum is similarly translated close to the harmonic frequencies. By fixing the working frequency higher than the hall sensor $1 / f$ corner frequency, the hybrid sensor could be used to remove the low bare hall sensor $1 / f$ noise as [9].

The expected performance of the system in terms of sensitivity and noise level are compared to those of a single Hall element (Table II). The sensor performance was deduced from experimental results.

To highlight the Hybrid YIG magnetic sensor noise performance, the equivalent magnetic noise spectral density is also given for a low frequency $(10 \mathrm{~Hz})$. For the hybrid YIG sensor, it results in a completely white output noise level, as detailed in [9]. So, its equivalent magnetic noise spectral density is given by

$$
b_{n}(f)=\frac{\sqrt{P S D}}{G} \frac{\mathrm{T}}{\sqrt{ } \mathrm{Hz}}
$$

in a wideband demodulation.

\section{CONCLUSION}

The demonstrated results clearly validate the working principle of the sensor, which can be used in order to simultaneously sense the two in-plane components of low-frequency magnetic applied field. Furthermore, it could be implemented on a single chip by using planar excitation coils [4].

Presently, the main improvement compared to bare Hall sensor is the reduction of the low-frequency noise. Unfortu- 
nately, the sensitivity improvement is mainly limited by the coupling factor. That parameter could be improved by reducing the distance ( $z$ axis) between the YIG thin-film surface and the Hall sensor. Meanwhile, difficulties appear in terms of the sensor position and spatial resolution that have to be taken into account [8], [9]. The ratio between the saturation magnetization $M_{S}$ and the magnetic field $H_{0}$ should also be investigated as a function of the YIG core geometry in order to increase the value of the hybrid sensitivity improvement term, $G$.

Finally, we demonstrated that the intrinsic magnetic hybrid sensor noise level is low. Results involve that better performance are achievable for this system if some significant optimizations are realized.

\section{ACKNOWLEDGMENT}

The authors thank I. Syvorotka, Scientific Research Company "Carat," Ukraine who offered the YIG samples for experiments and to V. Mosser, ITRON France, who provided nonencapsulated Hall device.

\section{REFERENCES}

[1] M. V. Valeiko, P. M. Vetoshko, A. Y. Perlov, and A. Y. Toporov, "Effect of the anisotropy constants on the magnetic susceptibility of materials with a cubic crystal structure," Phys. Solid State, vol. 36, no. 10, pp. 1631-1632, Oct. 1994.

[2] M. V. Valeiko, P. M. Vetoshko, and P. I. Nikitin, "Uniform rotation of magnetisation in thin magnetic films: Study of complex susceptibility and development of magnetometers," in Proc. 3rd EMSA Conf., 2000, pp. 141-142.

[3] H. Bittel, "Noise of ferromagnetic materials," IEEE Trans. Magn., vol. 5, pp. 359-365, 1969.

[4] C. Petridis, P. D. Dimitropoulos, and E. Hristoforou, "New magnetic field sensor based on combined flux-gate/Hall-effect arrangement," IEEE Sensors J., vol. 9, pp. 128-134, Feb. 2009.

[5] S. B. Ubizskii, "Orientational states of magnetization in (111)-oriented iron garnet films," J. Magn. Magn. Mat., vol. 195, pp. 575-582, 1999.
[6] C. Petridis, I. Petrou, P. Dimitropoulos, and E. Hristoforou, "Determining appropriate magnetic core properties for a new type of flux-gate like sensor," Sensor Lett., vol. 5, pp. 98-101, 2007.

[7] P. M. Vetoshko, M. V. Valeiko, and P. I. Nikitin, "Epitaxial yttrium iron garnet film as an active medium of an even-harmonic magnetic field transducer," Sens. Actuators A: Phys., vol. 106, pp. 270-273, 2003.

[8] C. Cordier, L. Méchin, C. Gunther, M. L. C. Sing, D. Bloyet, and V. Mosser, "Hall sensor response to an inhomogeneous magnetic field," IEEE Sensors J., vol. 5, no. 5, pp. 934-941, Oct. 2005.

[9] A. Qasimi, C. Dolabdjian, D. Bloyet, and V. Mosser, "Improvement of the $\mu$-Hall magnetic sensor sensitivity at low frequency," IEEE Sensors J., vol. 4, no. 1, pp. 160-166, Feb. 2004. 